\title{
Une délétion du gène de l'ankyrine à l'origine d'une forme de sphérocytose héréditaire
}

La sphérocytose héréditaire $(\mathrm{SH})$ est une des maladies hémolytiques les plus fréquentes. Sa transmission est, le plus souvent mais non toujours, autosomique dominante. Les hématies de ces sujets sont déficientes en spectrine (voir l'article récent de Delaunay et Dhermy dans $\mathrm{m} / \mathrm{s}$ [1]. Les gènes des chaînes $\alpha$ et $\beta$ de la spectrine sont portés respectivement par les chromosomes 1 et 14. Mais dans un certain nombre de cas la cause du déficit en spectrine reste mystérieuse. Il existe un autre locus génétique pour la $\mathrm{SH}$, dit $\mathrm{SPH} 2$. Il a été reconnu par des études familiales, siège sur le bras court du chromosome 8, alors que cette localisation n'était connue jusque-là pour aucune protéine de membrane du globule rouge. Or, en 1988, Coetzer et al. [2] avaient trouvé chez deux malades non apparentés atteints d'anémie hémolytique une baisse de $50 \%$ à la fois de la spectrine et d'une autre protéine de la membrane, l'ankyrine. Un groupe comprenant plusieurs équipes américaines (15 auteurs) a donc fait l'hypothèse que l'ankyrine, dont le clonage a été réalisé récemment, pouvait être en cause dans la SPH2 [3]. Un clone codant pour la partie C-terminale de la protéine a été hybridé à des chromosomes séparés à l'aide d'un trieur, et le gène a pu être assigné au chromosome 8 en 8p11.2. Cela concordait avec la position obtenue pour le locus SPH2 grâce à l'étude de familles portant des translocations équilibrées impliquant le chromosome 8 et des cas associés à des délétions interstitielles hétérozygotes entre $8 \mathrm{p} 11.1$ et 8 $\mathrm{p} 1122$, au voisinage du gène de la glutathion réductase, avec lequel il ne se confond cependant pas.

Pour tester l'hypothèse de l'identité entre le locus de l'ankyrine et celui de $\mathrm{m} / \mathrm{s} n^{\circ} 8$, vol. 6 , octobre 90
SPH2, les auteurs ont hybridé l'ADN de deux enfants non apparentés avec délétion du chromosome 8 à des sondes d'ankyrine, en prenant comme témoin interne une sonde d'une autre protéine de la membrane érythrocytaire, l'échangeur d'anions AE1. Le rapport des signaux est d'environ 0,5 chez les malades. Les résultats ont été confirmés en employant une méthode d'hybridation in situ en fluorescence, qui permet de voir directement chaque copie du gène sur son chromosome. Chez les malades on ne voit qu'un signal pour l'ankyrine, au lieu de deux chez les témoins, ainsi que pour le gène AE1. Enfin l'ankyrine a été dosée directement après électrophorèse, donnant chez un enfant atteint $59 \%$ du taux normal, mais aussi une baisse modérée de la spectrine et de la protéine 4,2 , en accord avec la liaison connue de ces deux protéines à l'ankyrine.

Un déficit hétérozygote en ankyrine dû à l'inactivation ou à la délétion d'un allèle paraît donc capable de provoquer une forme hémolytique de sphérocytose héréditaire. Il se pourrait que des déficits moins importants interviennent dans d'autres formes moins sévères de la maladie : on a retrouvé chez certains malades des taux modérément diminués à la fois de spectrine et d'ankyrine [3]. Des anomalies fonctionnelles pourraient également exister : on a décrit un polymorphisme de restriction du gène de l'ankyrine à l'enzyme NcoI, lié à un locus pour une $\mathrm{SH}$ dominante, avec déficit modéré en spectrine sans baisse de la quantité d'ankyrine ; l'interprétation - non démontrée est qu'une anomalie qualitative de l'ankyrine altérerait sa liaison avec la spectrine [3].

Il est enfin intéressant de signaler qu'un mutant chez la souris, appelé $\mathrm{nb}$, est atteint de normoblastose avec anémie hémolytique sévère et avec déficit de $50 \%$ en spectrine [4]. Ce mutant présente une molécule d'ankyrine anormale car tronquée (150 kDa au lieu de 250). Curieusement le locus de l'ankyrine comme de la normoblastose de la souris est strictement homologue de celui de l'ankyrine humaine, au voisinage du centromère sur le chromosome 8.

J.-C. D.

1. Delaunay J, Dhermy D. Les maladies génétiques du squelette érythrocytaire. médecine/sciences $1990 ; 6$ : 562-70.

2. Coetzer TL, Lauber J, Liu SC, et al. Partial ankyrin and spectrin deficiency in severe atypical hemolytic spherocytosis. $N$ Engl J Med 1988 : 318: 240-4.

3. Lux SE, Tse WT, Menninger JC, et al. Hereditary spherocytosis associated with deletion of human erythrocyte ankyrin gene on chromosome 8 . Nature 1990 ; 345 : 736-9.

4. White RA, Birkenmeier CS, Lux SE, Barker JE. Ankyrin and the hemolytic anemia mutation, nb, map to mouse chromosome 8: presence of the nb allele is associated with a truncated erythrocyte ankyrin. Proc Natl Acad Sci USA 1990; 87 : 3117-21.
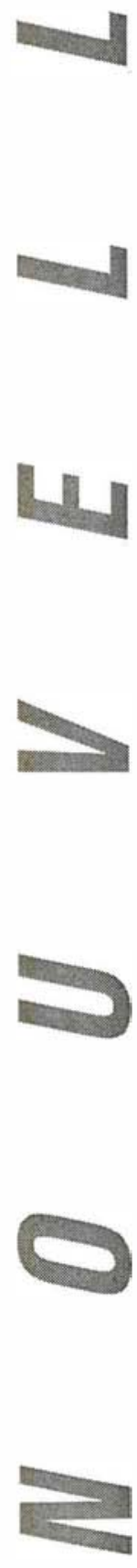